\title{
Increasing Access to Hepatitis C Virus Medications: A Program Model Using Patient Navigators and Specialty Pharmacy to Obtain Prior Authorization Approval
}

\author{
Trang M. Vu, MD; Wilma Toribio, MD; Farah Riazi, MD; Genesis Ciprian, BA; Nathalia Gibbs, BS; \\ Martha Giardina, RN; Jocelyn A. Camacho, MPH; Korin Parrella, MPH; Joy Cambe, MD, MPH; \\ Catherine Amory, LCSW; Rachel Chasan, MD; Keith M. Sigel, MD, MPH; and Jeffrey J. Weiss, PhD, MS
}

\begin{abstract}
BACKGROUND: Obtaining prior authorization (PA) approval for the new direct-acting antiviral (DAA) hepatitis $C$ medications is time consuming and requires specific expertise. Our primary care-based program treats hepatitis $\mathrm{C}$ virus (HCV)-infected patients at an urban academic medical center and employs patient navigators trained in the PA process who collaborate with a nurse and specialty pharmacy to manage the PA process.
\end{abstract}

OBJECTIVE: To demonstrate the rate of PA approvals for our programmatic model and determine potential predictors of PA approval.

METHODS: We conducted a review of program databases and medical records of patients for whom DAA hepatitis $C$ medications were ordered between November 1, 2014, and October 31, $2015(n=197)$. We first evaluated patient characteristics associated with the number of steps to approval. Then we used a multivariable ordinal regression to determine independent predictors of fewer steps to approval. Using Kaplan-Meier methods, we assessed patient characteristics associated with approval time and then fit a multivariable Cox regression model to determine independent predictors of time to approval.

RESULTS: Of the 197 patients, $69 \%(n=136)$ had Medicaid; $12 \%(n=24)$ had Medicare; $10 \%(n=19)$ had both Medicaid and Medicare; $5 \%(n=10)$ had private insurance; and $4 \%(n=8)$ were uninsured. Ninety-three percent of the patients were eventually approved for HCV treatment. The steps in the PA cascade were approval on first submission ( $37 \%$; mean days $=30.7$; $\mathrm{SD}=29.9)$; approval after internal appeal $(45 \%$; mean days $=66.8 ; \mathrm{SD}=$ 70.5); approval after external appeal (11\%; mean days $=124.7$; $S D=60.2)$; and no approval obtained (7\%). Unadjusted factors found to have a $P$ value $<0.200$ in relation to fewer steps in the PA cascade were older age, female gender, non-Medicaid insurance, comorbid hypertension, comorbid diabetes, being domiciled, and being nongenotype 2 . After adjustment, nonMedicaid insurance and nongenotype 2 remained significant. In survival analysis, non-Medicaid insurance and mid-range fibrosis were associated with fewer days to PA approval.

CONCLUSIONS: Our program obtained $93 \%$ of PA approvals for hepatitis C medications. Patient navigators collaborating with a nurse and specialty pharmacy as a program may improve the PA approval process, although further research with a control group is necessary.

J Manag Care Spec Pharm. 2018;24(4):329-33

Copyright $\odot 2018$, Academy of Managed Care Pharmacy. All rights reserved.

\section{What is already known about this subject}

Although the new direct-acting antiviral (DAA) medications for treating hepatitis $\mathrm{C}$ virus ( $\mathrm{HCV}$ ) infection have proven to be more effective and tolerable, the increased cost of the medications has resulted in nearly all insurers implementing prior authorization (PA) requirements to obtain access

Successfully obtaining approval for hepatitis C medications through the PA process requires specific expertise, as individual insurers have different policies for determining which patients qualify for treatment.

The PA process is time consuming for providers and patients and has become a major barrier to access to treatment for HCV infections.

\section{What this study adds}

This study describes a programmatic model of skilled patient navigators trained in the PA process working with a nurse and a specialty pharmacy to obtain PA approvals for DAA medications for HCV infection in an urban primary care clinic.

PA approvals were obtained for 93\% of DAA hepatitis C drug prescriptions written during the 1-year period between November 1 2014, and October 31, 2015.

In multivariate analysis, non-Medicare insurance and genotype 2 were associated with the most steps to PA approval. Medicare insurance and mid-range fibrosis staging were associated with the shortest time to PA approval.

$\mathrm{T}$ he high costs of direct-acting antivirals (DAAs) to treat hepatitis $\mathrm{C}$ virus (HCV) infection have led to insurers requiring DAA prescriptions undergo prior authorization (PA), a process by which a health insurance provider determines if a specific treatment is medically necessary and will allow for insurance coverage of the treatment cost or will potentially consider cost-effective alternatives. ${ }^{1}$ Successfully obtaining approval for HCV medications through the PA process requires specific expertise, as individual insurers have different policies for determining which patients qualify for all-oral DAA treatments. Although evidence suggests that early treatment of HCV infection is cost-effective, ${ }^{2}$ many insurers, 
including Medicaid, limit access to patients who meet certain requirements, such as those with advanced liver fibrosis. ${ }^{3-5}$ The PA process is time-consuming for providers, patients, and payers and has become a major barrier to access to treatment for $\mathrm{HCV}$ infection. ${ }^{6}$

The Respectful \& Equitable Access to Comprehensive Healthcare (REACH) program based within the primary care clinic at Mount Sinai Hospital in New York City uses a multidisciplinary team model to provide medical, mental health, social work, and patient navigation services to HCV-infected patients with complex medical and psychiatric profiles. ${ }^{7}$ It is also one of New York State Department of Health's funded sites to provide care to uninsured patients under the Hepatitis C Patient Assistance Program (HepCAP). The program addresses all steps in the HCV cascade of care, including communitybased testing, linkage to care, retention in care, treatment readiness, and reducing barriers to successful treatment completion and adherence. Our program utilizes patient navigators working with a nurse and 1 specialty pharmacy to manage the PA process, lessening the burden on physicians and patients.

Previous studies have reported $81 \%-91 \%$ of patients get insurance approval for therapy. ${ }^{1,4,8}$ In general, patients with advanced liver disease and Medicare were more likely to be approved. ${ }^{4,8}$ Conversely, Medicaid insurance was more likely to be associated with medication denials. ${ }^{8}$ Thus far, however, no program has demonstrated the efficacy of patient navigators working with a specialty pharmacy in obtaining medication approvals. Our study aim is to examine the number of successful PA approvals obtained in our program and to analyze specific factors that may predict a PA being approved.

\section{Methods}

Approval from the Mount Sinai Institutional Review Board (IRB) was obtained to conduct a retrospective chart review of program databases and medical records of all patients in the REACH program for whom DAA HCV medications were ordered in the 1-year period between November 1, 2014, and October 31, 2015 ( $\mathrm{n}=197)$. Patients were followed for 180 days or until PA approval was obtained, whichever came earlier. We examined the relationship between patient clinical characteristics and (a) the number of steps in the PA process and (b) the time to medication approval.

All DAA HCV medication prescriptions from the REACH program were sent to 1 specialty pharmacy (AcariaHealth) initially. In a small number of cases, prescriptions were then referred to a different specialty pharmacy only when required by the insurance company. Once a DAA HCV medication was prescribed, the program nurse collected documentation required by the insurer, such as baseline laboratory results, fibrosis staging, provider clinic notes, medical necessity letter, evidence of patient's readiness to start treatment, and (depending on the insurance company) a urine drug screen. The nurse then sent the collated information to the specialty pharmacy to begin processing the PA. The patient navigators then communicated with the specialty pharmacy's PA office and insurance companies through fax and phone calls if further information was required, submitted paper work for appeal submissions as necessary, and advocated for the patient until the PA process was successful.

Demographic and clinical data collected included age, gender, race/ethnicity, insurance, HCV genotype, liver fibrosis staging by Fibrosis-4 (FIB-4) score, ${ }^{9}$ psychiatric history, history of HCV treatment, DAA regimen prescribed, date PA was sent to specialty pharmacy, date internal appeal was initiated after first adverse determination, date external appeal was initiated after final determination from insurance, and date medication approval was received. Data were collected from electronic medical records and from an existing internal tracking database that was managed by the patient navigators. We also collected information on the date the prescription was sent to patient assistance programs of pharmaceutical companies to cover medications for uninsured patients or patients whose insurance would not cover DAA therapy. Insurance was classified as Medicaid (with no differentiation made between feefor-service or managed care plans), Medicare, dual (patients who had both Medicaid and Medicare), private (which included commercial insurance through the Affordable Care Act and/or employer), and uninsured. We calculated the time in days and number of steps that it took from the first submission of a PA until approval: 1 step signified approval achieved after initial submission to specialty pharmacy or patient assistance program; 2 steps signified approval after submission of an internal appeal or to patient assistance program after insurance denial; and 3 steps signified approval after submission of external appeal or patient assistance program after insurance final denial. All appeals were submitted within 10 business days of receiving the denial letter.

Our primary outcome of interest was an ordinal variable representing the number of steps in the medication approval process required to obtain PA approval; the secondary outcome measure was the time needed to obtain medication approval in days. We ascertained potential factors influencing this process including demographics, patient insurance type, comorbid illnesses, and stage of liver disease (as measured by FIB-4 scores). In univariate analyses, we evaluated patient characteristics associated with the number of steps in the approval process and we then entered variables with $P$ values $<0.200$ in a multivariable ordinal logistic regression to determine independent predictors of fewer steps in the drug approval process. Using Kaplan-Meier methods, we conducted univariate analyses that evaluated patient characteristics associated with approval time (up to 180 days) and then fit a multivariable Cox regression model using variables with $P$ values $<0.200$ to determine independent predictors of time to approval by 180 days. 


\begin{tabular}{|c|c|}
\hline Characteristic & Frequency $(\mathrm{N}=197)$ \\
\hline Mean age in years $+\mathrm{SD}$ & $56.8+10.6$ \\
\hline \multicolumn{2}{|l|}{ Gender, n (\%) } \\
\hline Female & $74 \quad(37.6)$ \\
\hline Male & $123 \quad(62.4)$ \\
\hline \multicolumn{2}{|l|}{ Race, n (\%) } \\
\hline Asian & (1.5) \\
\hline Black & $72 \quad(36.5)$ \\
\hline Hispanic & $74 \quad(37.6)$ \\
\hline White & $43 \quad(21.8)$ \\
\hline Other & $3 \quad(1.5)$ \\
\hline Unknown & $(1.0)$ \\
\hline \multicolumn{2}{|l|}{ Insurance type, n (\%) } \\
\hline Medicaid & $136 \quad(69.0)$ \\
\hline Medicare & $24 \quad(12.2)$ \\
\hline Medicaid/Medicare & $19 \quad(9.6)$ \\
\hline Private & $10 \quad(5.1)$ \\
\hline Uninsured & $8 \quad(4.1)$ \\
\hline \multicolumn{2}{|l|}{ Genotype, n (\%) } \\
\hline $1(a+b)$ & $170 \quad(86.3)$ \\
\hline 2 & $13 \quad(6.6)$ \\
\hline 3 & $11 \quad(5.6)$ \\
\hline 4 & $(1.0)$ \\
\hline 6 & $1 \quad(0.5)$ \\
\hline \multicolumn{2}{|l|}{ FIB-4 stage, n (\%) } \\
\hline 1 (FIB-4 score < 1.45$)$ & $60 \quad(30.5)$ \\
\hline 2 (FIB-4 score 1.45-3.25) & $97 \quad(49.2)$ \\
\hline 3 (FIB-4 score $>3.25$ ) & $40 \quad(20.3)$ \\
\hline \multicolumn{2}{|l|}{ Prior HCV treatment, $\mathrm{n}(\%)$} \\
\hline No & $155 \quad(78.7)$ \\
\hline Yes & $42 \quad(21.3)$ \\
\hline \multicolumn{2}{|l|}{ Homelessness, n (\%) } \\
\hline No & $186 \quad(94.4)$ \\
\hline Yes & $11 \quad(5.6)$ \\
\hline \multicolumn{2}{|l|}{ Comorbid psychiatric diagnosis, $\mathrm{n}(\%)$} \\
\hline No & $116 \quad(58.9)$ \\
\hline Yes & $81 \quad(41.1)$ \\
\hline \multicolumn{2}{|l|}{ Comorbid renal diagnosis, $\mathrm{n}(\%)$} \\
\hline No & $190 \quad(96.4)$ \\
\hline Yes & $7 \quad(3.6)$ \\
\hline \multicolumn{2}{|l|}{ Comorbid diabetes mellitus diagnosis, n (\%) } \\
\hline No & $153(77.7)$ \\
\hline Yes & $44 \quad(22.3)$ \\
\hline \multicolumn{2}{|l|}{ Comorbid hypertension diagnosis, $\mathrm{n}(\%)$} \\
\hline No & $122(61.9)$ \\
\hline Yes & $75 \quad(38.1)$ \\
\hline \multicolumn{2}{|l|}{ Regimen prescribed, n (\%) } \\
\hline Ledispavir/sofosbuvir & $166 \quad(84.3)$ \\
\hline Sofosbuvir + ribavirin & $14 \quad(7.1)$ \\
\hline Sofosbuvir+daclatasvir & $10 \quad(5.1)$ \\
\hline Sofosbuvir+simeprevir & $4 \quad(2.0)$ \\
\hline Ledipasvir/sofosbuvir + ribavirin & $2 \quad(1.0)$ \\
\hline Ombitasvir/paritaprevir/ritonavir/dasabuvir & $1 \quad(0.5)$ \\
\hline
\end{tabular}

\section{Results}

Patients in this study $(\mathrm{n}=197)$ were insured by Medicaid $(69 \%$, $\mathrm{n}=136)$, Medicare $(12 \%, \mathrm{n}=24)$, both Medicaid/Medicare $(10 \%$, $\mathrm{n}=19)$, or private insurance $(5 \%, \mathrm{n}=10)$ or were uninsured $(4 \%, n=9)$. They were predominantly male $(62 \%, n=123)$ with a mean age of 56.8 years (standard deviation $[S D]=10.6$ ), were mostly Hispanic $(38 \%, \mathrm{n}=74)$ or black $(36 \%, \mathrm{n}=72)$, were largely treatment naive $(79 \%, \mathrm{n}=155)$, and had mostly genotype $1(86 \%, n=170 ;$ Table 1$)$. The medications applied for were ledipasvir/sofosbuvir $(84.3 \%, \mathrm{n}=166)$, sofosbuvir and ribavirin $(7.1 \%, \mathrm{n}=14)$, sofosbuvir and daclatasvir $(5.1 \%, \mathrm{n}=10)$, sofosbuvir and simeprevir $(2.0 \%, \mathrm{n}=4)$, ledipasvir/sofosbuvir and ribavirin ( $1 \%, \mathrm{n}=2)$, and ombitasvir/paritaprevir/ritonavir/ dasabuvir $(0.5 \%, \mathrm{n}=1)$.

Our program obtained HCV medication approval for $93 \%$ (95\% confidence interval $[\mathrm{CI}]=88 \%-96 \%)$ of patients. The mean time to approval was 59.8 days $(S D=64.5)$. The steps in the PA cascade were approval on first submission (1 step; $37 \%$; mean days $=30.7 ; S D=29.9$, approval after internal appeal (2 steps; 45\%; mean days $=66.8 ; \mathrm{SD}=70.5$ ), approval after external appeal (3 steps; 11\%; mean days $=124.7$; $\mathrm{SD}=60.2$ ), and no approval obtained as of May $2016(7 \%, \mathrm{n}=13$; Figure 1). Among those patients for whom no approval was obtained, 9 were lost to follow-up, 2 missed the appeal period, 1 had insurance that became inactive, and 1 decided not to pursue treatment after an external appeal was denied. Medications were obtained through pharmaceutical assistance programs for all 8 patients treated through the HepCAP program for uninsured residents of New York State.

Demographic and clinical variables were examined for univariate associations with steps in the medication approval process for patients with medical insurance $(n=189)$. In univariate analysis, those factors found to have a $P$ value $<0.200$ in relation to fewer steps in the PA cascade were older age $(P=0.138)$, non-Medicaid insurance $(P=0.001)$, comorbid hypertension $(P=0.094)$, comorbid diabetes $(P=0.042)$, being domiciled $(P=0.046)$, and genotype $1 \quad(P=0.084)$. Fibrosis stage as determined by the FIB-4 score was not statistically significantly associated with medication approval. Our adjusted logistic model showed that Medicare (odds ratio $[\mathrm{OR}]=0.215$; 95\% CI $=0.797-0.580)$ or Medicaid/Medicare (OR $=0.38$; 95\% $\mathrm{CI}=0.144-0.984)$ insurance compared with Medicaid resulted in fewer steps in the PA cascade. There was no difference for private insurance versus Medicaid $(\mathrm{OR}=0.502 ; 95 \% \mathrm{CI}=0.133$ 1.890). In addition, genotype 2 resulted in more steps in the PA cascade $(\mathrm{OR}=2.998 ; 95 \% \mathrm{CI}=1.068-8.418)$ compared with genotype 1 (no difference for genotype 3 [OR $=0.698$; 95\% $\mathrm{CI}=0.202-2.411] \quad$ or $4 \quad[\mathrm{OR}=1.388 ; 95 \% \mathrm{CI}=0.104-18.582]$ compared with genotype 1 )

In a time-to-event analysis with medication approval as the primary outcome (subjects were censored if not receiving medication by the 180th day), the variables that had a 


\section{FIGURE 1 Flowchart of Approvals and Denials for Patients with Number of Steps in} Prior Authorization Process

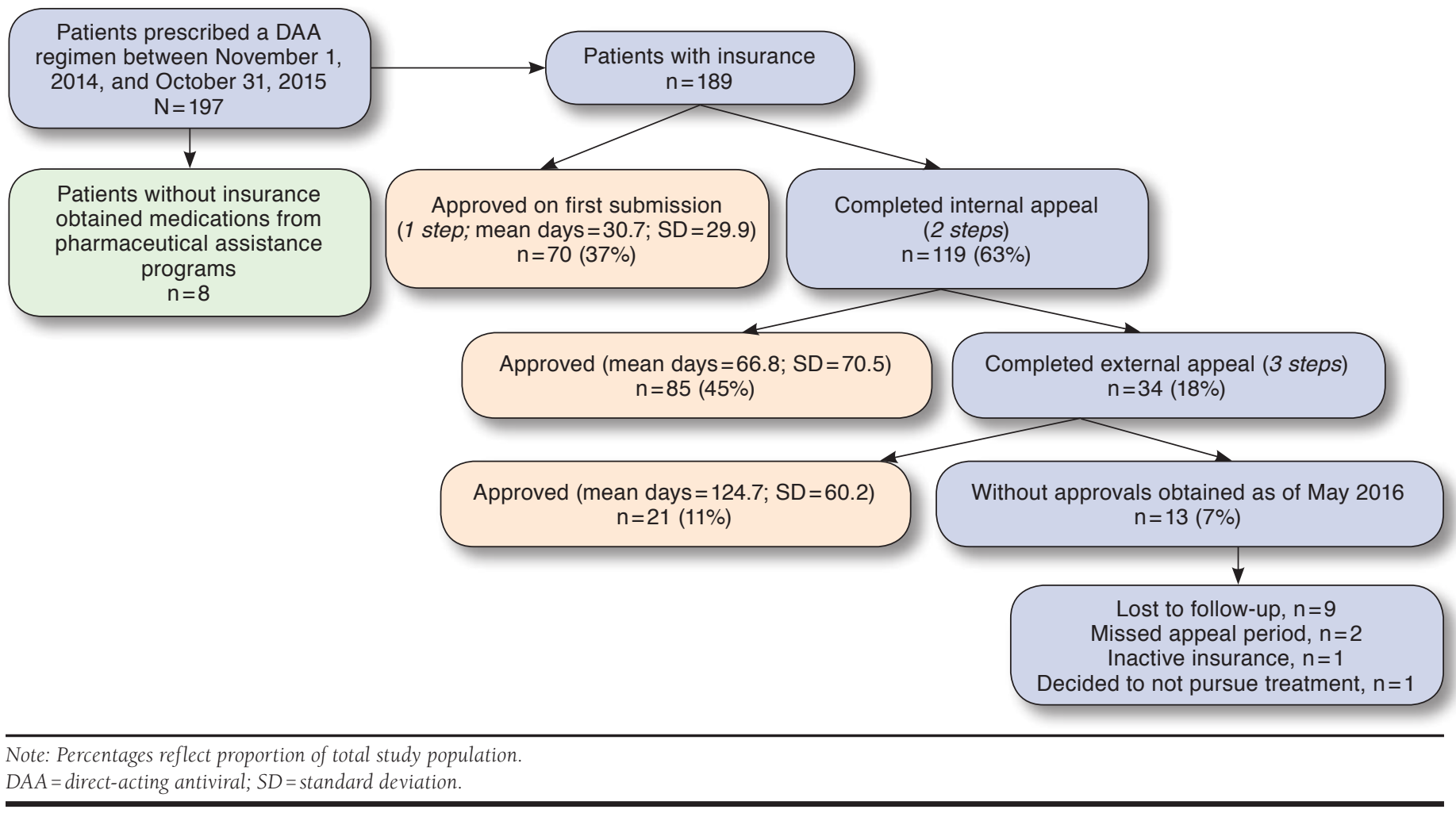

$P$ value $<0.200$ in relation to this outcome were insurance type $(P \leq 0.000)$, hypertension $(P=0.127)$, and FIB-4 score $(P=0.010)$. In an adjusted analysis (Cox regression) including age and sex, only insurance type (hazard ratio $[\mathrm{HR}]=3.143$; $95 \% \mathrm{CI}=1.871-5.279 ; P \leq 0.000)$ and disease stage $(\mathrm{HR}=0.575$; $95 \% \mathrm{CI}=0.391-0.846 ; P=0.005)$ retained significance. Those with Medicare (median $=14.0$ days; $95 \% \mathrm{CI}=4.43-23.57$ ) or dual Medicare/Medicaid insurance (median $=19.0$ days; 95\% $\mathrm{CI}=7.40-30.60)$ took significantly less time for approval than those with Medicaid (median $=50.0$ days; 95\% CI $=42.86$ 57.14; $P=0.022$ and $P=0.001$, respectively). Furthermore, those with the highest fibrosis scores (FIB-4 range $>3.25$ ) had slower medication approval (mean $=83.6$ days; standard error $[S E]=10.5)$ than those with midrange fibrosis scores (FIB-4 range $=1.45-3.25$; mean $=52.3$ days; $S E=5.2 ; P=0.005$ ).

\section{Discussion}

In this study of an integrated program model of patient navigators working in conjunction with a nurse and a specialty pharmacy, 93\% of chronic HCV-infected patients were approved for HCV medication for whom the initial PA was submitted in the period between November 1, 2014, and October 31, 2015.
Our program's ability to obtain approvals for 93\% of patients is slightly higher than the approval rates published in previous studies, which have ranged from $81 \%-91 \% .{ }^{1,3,4}$ To our knowledge, this is the first study demonstrating the role of patient navigators and a nurse coordinating PAs with a specialty pharmacy. Our program's high rate of medication approval suggests that experienced patient navigators working in collaboration with a nurse and a specialty pharmacy can be an alternative model for obtaining PA approvals. Further research is needed to evaluate the cost-effectiveness of this model compared with other models of care that do not utilize patient navigators. ${ }^{10}$

In our urban HCV treatment program, Medicaid insurance and genotype 2 were independently associated with increased steps to HCV medication approval. Medicare insurance and mid-range fibrosis staging were independently associated with less time to PA approval. The identification of Medicaid insurance as a barrier to PA authorization is consistent with other studies. ${ }^{4,8}$ It also suggests that PA requirements for the DAA regimens are similarly restrictive across different state Medicaid plans. The genotype 2 finding is likely related to the specific medications ordered for this genotype (sofosbuvir and ribavirin; sofosbuvir and daclatasvir). 
The finding that patients with midrange fibrosis scores had faster medication approvals than those with advanced fibrosis was unexpected. Previous studies have demonstrated that patients with advanced fibrosis resulted in higher likelihood of medication approval ${ }^{4,8}$ and faster time to approval. During our study period, however, the New York State Attorney General reached an agreement with 7 major commercial health insurance companies in New York State that eliminated restrictions to coverage such as advanced liver disease. ${ }^{11}$ This likely had a major effect on DAA approvals for our patient population.

\section{Limitations}

This study has some limitations to consider. One limitation is the lack of a control group to which we can compare the rate of PA approvals. Without a control group, we cannot definitively attribute the high approval rate obtained from our clinic as being due to the work of the patient navigators, nurse, and specialty pharmacy. However, if previous studies, which did not utilize patient navigators, can be seen as historical controls, then our higher rate of PA approvals suggests that patient navigators may increase the rate of PA approvals., ${ }^{1,3,4}$ Other limitations include the lack of generalizability of our results to other states, as the New York State Medicaid insurance plan restrictions may vary from other states. Our study has a very small number of privately insured patients, so the findings cannot be generalized to this population. Additionally, the study was conducted in a large tertiary care academic medical center and findings may not be applicable to other types of health care facilities, including community-based clinics.

\section{Conclusions}

The REACH program obtained PA approvals for 93\% of HCV medication prescriptions during the period between November 1 , 2014, and October 31, 2015. Insurance status was 1 factor identified as determining the amount of work required (i.e., number of steps), as was time required to obtain PA approval. The mean time to approval for all patients was approximately 2 months.

\section{Authors}

TRANG M. VU, MD; WILMA TORIBIO, MD; FARAH RIAZI, MD; GENESIS CIPRIAN, BA; NATHALIA GIBBS, BS; MARTHA GIARDINA, RN; JOCELYN A. CAMACHO, MPH; KORIN PARRELLA, MPH; JOY CAMBE, MD, MPH; CATHERINE AMORY, LCSW; RACHEL CHASAN, MD; KEITH M. SIGEL, MD, MPH; and JEFFREY J. WEISS, PhD, MS, Division of General Internal Medicine, Icahn School of Medicine at Mount Sinai, New York, New York.

AUTHOR CORRESPONDENCE: Trang M. Vu, MD, Assistant Professor, Division of General Internal Medicine, Icahn School of Medicine at Mount Sinai, Center for Advanced Medicine,

17 E. 102nd St., 6th Fl. West, New York, NY 10029

Tel.: 212.824.7433; E-mail: trang.vu@mountsinai.org.

\section{DISCLOSURES}

The Respectful \& Equitable Access to Comprehensive Healthcare (REACH) program receives funding from the Robin Hood Foundation and the New York State Department of Health AIDS Institute. Weiss receives grant support from Gilead Sciences and has served as a consultant for AbbVie and Gilead Sciences. Vu reports speaker fees from Peer View Institute. All other authors report no conflict of interest.

Study design and concept were contributed by Chasan, Sigel, Vu, and Weiss. Riazi, Ciprian, Giardina, and Gibbs collected the data, which were interpreted by Toribio, Amory, Chasan, and Sigel. The manuscript was written by Vu and Weiss and revised by Parrella, Cambe, Camacho, and Vu.

Research from this study was presented as an abstract poster on November 14, 2016, at the AASLD Liver Meeting in Boston, Massachusetts.

\section{REFERENCES}

1. Do A, Mittal Y, Liapakis A, et al. Drug authorization for sofosbuvir/ledipasvir (Harvoni) for chronic HCV infection in a real-world cohort: a new barrier in the HCV care cascade. PLoS One. 2015;10(8):e135645.

2. Chhatwal J, Kanwal F, Roberts MS, Dunn MA. Cost-effectiveness and budget impact of hepatitis $C$ virus treatment with sofosbuvir and ledipasvir in the United States. Ann Intern Med. 2015;162(6):397-406.

3. Younossi ZM, Bacon BR, Dieterich DT, et al. Disparate access to treatment regimens in chronic hepatitis $C$ patients: data from the TRIO network. J Viral Hepat. 2016;23(6):447-54.

4. Saab S, Jimenez M, Fong T, et al. Accessibility to oral antiviral therapy for patients with chronic hepatitis $C$ in the United States. J Clin Trans Hepatol. 2016;4(2):76-82

5. Clements KM, Clark RE, Lavitas P, et al. Access to new medications for hepatitis C for Medicaid members: a retrospective cohort study. J Manag Care Spec Pharm. 2016;22(6):714-22b. Available at: https://www.jmcp.org/ doi/10.18553/jmcp.2016.22.6.714.

6. Edlin BR. Access to treatment for hepatitis $C$ virus infection: time to put patients first. Lancet Infect Dis. 2016;16(9):e196-201.

7. Woodrell C, Weiss J, Branch A, Gardenier D, et al. Primary care-based hepatitis $C$ treatment outcomes with first-generation direct-acting agents. J Addict Med. 2015;9(5):405-10.

8. Lo Re V 3rd, Gowda C, Urick PN, et al. Disparities in absolute denial of modern hepatitis $\mathrm{C}$ therapy by type of insurance. Clin Gastroenterol Hepatol. 2016;14(7):1035-43.

9. Sterling RK, Lissen E, Clumeck N, Sola R, et al. Development of a simple noninvasive index to predict significant fibrosis in patients with HIV/HCV coinfection. Hepatology. 2006;43(6):1317-25.

10. Martin MT, Telebak E, Taylor PA, Volozhina O. Development of a specialty medication prior-authorization service at an urban academic medical center. Am J Health Syst Pharm. 2016;73(15):1174-79.

11. New York State Office of the Attorney General. Press Release. A.G. Schneiderman announces major agreement with seven insurers to expand coverage of chronic hepatitis $C$ treatment for nearly all commercial health insurance plans across New York State. April 26, 2016. Available at: https:// ag.ny.gov/press-release/ag-schneiderman-announces-major-agreement-seveninsurers-expand-coverage-chronic. Accessed March 9, 2018. 